\title{
1 Jahr Psychotherapieverordnung = Vernichtung von 10 Mannjahren Arbeit
}

Heiner Lachenmeier

Korrespondenz:

Dr. med. Heiner Lachenmeier Zürichstrasse 49

CH-8910 Affoltern am Albis

hlachenmeier@hin.ch

\section{Ausgangslage}

Ein Internist und Master in Public Health in Diensten des Bundes will die 50 Milliarden Gesundheitskosten senken. Mit «träfer» Klarsicht sieht er grosses Sparpotential bei der Psychotherapie, einem Teilbereich der ambulanten Psychiatrie, die alles eingeschlossen mit rund 500 Millionen gerade mal ein Prozent der Kosten ausmacht.

Soweit die mathematischen Voraussetzungen. Als klar wird, dass Sparpotential schon rein mathematisch kein Argument sein kann, wechselt die Argumentation in Richtung Verbesserung der Verteilung der anerkannt zu geringen therapeutischen Ressourcen.

Seit einem Jahr nun müssen neu begonnene Psychotherapien spätestens nach sechs Sitzungen gemeldet werden. Die Vertrauensärzte der Krankenkassen entscheiden aufgrund der eingereichten Formulare, ob die Kostengutsprache für die Psychotherapie gesprochen wird.

\section{Überschlagsrechnung 2007}

- Grössenordnung 2000 meldende Ärzte (Psychiater sowie Somatiker, die Psychotherapie delegieren);

- Minimum 10 meldepflichtige Therapien pro Arzt (inklusive deren delegiert arbeitender Psychologen);

- insgesamt 1 Stunde verbrauchte Arbeitszeit pro Meldung, aufgeteilt in:

- 1/4 Std. Aufwand Vorbereitung, Formular ausfüllen, abschicken, Leistung erfassen,

- 1/4 Std. Besprechung Formular mit Patient,

- 1/4 Std. Aufwand Vertrauensarzt (falls er das Formular tatsächlich studiert und abwägende Gedanken macht),

- 1/4 Std. Aufwand nach dem Entscheid wie Administrationsaufwand Krankenkasse mit Registrierung, Rückmeldung an Arzt sowie Administrationsaufwand beim Arzt nach der Rückmeldung,

(- unbekannter Zeitaufwand durch wiederkehrendes Kopfschütteln über das Ausmass an Unsinn).

Das ergibt 20000 aufgewendete Stunden (etwa hälftig auf behandelnde Ärzte und Krankenkassen verteilt).
Nach einem Sicherheitsabzug von 10\% sind es noch 18000 Stunden. Rechnen wir mit einer (im gesellschaftlichen Vergleich überdurchschnittlichen) Jahresarbeitszeit von 1800 Stunden, dann bleibt die Grössenordnung von 10 Mannjahren vernichteter produktiver Arbeitsleistung.

Schon im Sommer 2007 berichteten die Krankenkassen aufgrund der Realerfahrungen in der Umsetzung, dass die neue Verordnung nichts bringt.

\section{Gesetz und Verordnung}

Das Bundesgesetz über die Krankenversicherung (KVG) bestimmt in Art. 32, dass Leistungen (also auch die verlangten, nach KVG abzurechnenden Meldeformulare) wirksam, zweckmässig und wirtschaftlich sein müssen. Die Wirksamkeit muss nach wissenschaftlichen Methoden nachgewiesen sein.

Nun, die neuen Artikel 2 und 3 der Krankenpflege-Leistungsverordnung ...

W haben offensichtlich zero therapeutische Wirksamkeit, weder für die Patienten noch für das Gesundheitswesen;

können selbst bei bestem Willen keine Wissenschaftlichkeit für sich beanspruchen.

Begründung

- Nach 6 Sitzungen ist in der Regel noch keine fundierte Einschätzung eines Patienten möglich;

- die geforderten Angaben teilen sich wie folgt auf:

- 31/2 Zeilen: Beschreibung der Beschwerden und Symptome,

- 1/4 Zeile: Diagnose(n),

- 3 Zeilen: wichtige Zusatzinformationen,

- 33/4 Zeilen: Ziel und Zweck der Behandlung,

- 3/4 Zeile: Modalitäten der Therapie;

- aufgrund solch minimaler Informationen ist auch dem besten Facharzt für Psychiatrie und Psychotherapie keine fundierte Einschätzung der korrekten Indikationsstellung einer Psychotherapie möglich geschweige denn der Mehrheit der Vertrauensärzte, die über keine psychiatrischpsychotherapeutische Ausbildung verfügt; 
- natürlich dürfte man mehr schreiben, doch genügend gesicherte Information wäre zu diesem Zeitpunkt einer Behandlung meist gar nicht vorhanden. Und selbst wenn, wäre es surreal und sicher nicht wirtschaftlich, für das Honorar von Fr. 28.- noch mehr Zeit zu verschwenden;

- vorsichtig formuliert darf unter dem gegebenen Studiendesign - das Einkommen der Ausfüllenden hängt von deren Formulareinträgen $\mathrm{ab}$ - ein Fragezeichen hinter die Datenqualität gesetzt werden;

- laut Vorgaben genügt der Einsatz von Medikamenten nicht als Qualifizierung zur nichtmeldepflichtigen «integriert psychiatrisch-psychotherapeutischen Behandlung». Anders ausgedrückt: Aus Sicht des BAG müssen neuerdings Psychopharmaka als Psychotherapie betrachtet werden ...

- eine Studie mit diesem Design gilt zumindest in der Psychiatrie/Psychotherapie als unwissenschaftlich und würde niemals für EBM beigezogen.

Z haben sich als absolut zwecklos punkto bessere Verteilung der therapeutischen Ressourcen erwiesen, vielmehr haben sie mit 10000 Stunden ärztlicher Einsatzzeit die eh schon zu knappen Ressourcen um das Äquivalent von fünf Psychiatern geplündert.

W Weder in bezug auf Kostenersparnis noch in bezug auf Verteilung der therapeutischen Ressourcen dürfte es als wirtschaftlich gelten, wenn die Massnahme in beiden Bereichen das blanke Gegenteil vom angestrebten Ziel bewirkt und dafür pro Jahr 10 Mannjahre vergeudeter Arbeitszeit zu finanzieren sind. Immerhin wird - aus Sicht der Politik - verständlich, warum der Gesetzgeber nur bei der Wirksamkeit den wissenschaftlichen Nachweis verlangt, nicht aber bei der Wirtschaftlichkeit ...

\section{Auf den Punkt gebracht}

Die neuen Artikel 2 und 3 der KLV ...

- erfüllen nicht nur kein einziges der berühmten WZW-Kriterien, sondern gefährden gar durch ernsthafte Nebenwirkungen die Gesundung;

- laufen dem Art. 32 des KVG - also der hierarchischen höheren, rechtlichen Ebene - zuwider;

- können folglich als gesetzeswidrig bezeichnet werden.

$\mathrm{Zu}$ Recht hätte keine ärztliche Leistung und kein Medikament bei solch lausiger Qualität die Chance, als Pflichtleistung aufgenommen zu werden.

\section{Das Versagen der Verbände}

Angesichts des Ausmasses an Unsinn ist kaum zu widerlegen: Auch unsere eigenen Verbände haben kläglich versagt.

\section{Osteoporose der verschiedenen Verbandswirbelsäulen?}

FMH und psychiatrische Fachgesellschaften haben es nicht gewagt, sich einem fachlich und ökonomisch falschen sowie rechtswidrigen Projekt zu verweigern. Sie haben damit nicht nur das Vertrauen ihrer Mitglieder untergraben, sondern auch das Vertrauen der Öffentlichkeit in die zu Recht erwartete Wissenschaftlichkeit und Verlässlichkeit der Ärzteschaft.

\section{Strategische Blindheit}

Die strategische Bedeutung dieser neuen Verordnung für die gesamte Ärzteschaft wurde völlig ignoriert: Erstmals wird für einen ganzen Bereich der ambulanten Pflichtleistungen eine flächendeckende Pflicht für das Einholen einer Kostengutsprache bereits in der Anfangsphase der Behandlung eingefordert.

Die Pflicht wurde von der Vergütungspflicht der Kassen auf die Begründungspflicht der Ärzteschaft verschoben. Und das alles ohne auch nur eine ernstzunehmende Gegenmassnahme unserer Verbände - die Salami ist angeschnitten.

\section{Fazit}

Aufgrund der gegebenen Sachlage sollte erwartet werden können, dass zwischen den neuen Artikeln 2 und 3 der KLV und dem Bund das gleiche möglich ist wie schon zwischen dem Bund und dem eingangs erwähnten Initianten dieser Artikel: eine schnelle Trennung in gegenseitigem Einvernehmen.

\section{PS}

Alle sonst vorgebrachten Argumente wie Eingriff in die Behandlung, Datenschutzproblematik, Geringschätzung der psychiatrischen Patienten, des Fachs und der Fachärzte usw. gelten selbstverständlich auch.

Als Ärzte sollten wir freilich gelernt haben, in der Sprache der Patienten zu reden.

Die Patienten hier sind die Politik, die Verwaltung und gewisse Gesundheitsökonomen. Deren Sprache besteht vorwiegend aus Taktieren, Nutzung des Rechtsweges, medialen Elementen und Machtausübung. Nur zu einem kleinen Teil aus Vernunft. Wenn wir politisch (be-)handeln wollen, müssen wir diese Sprache lernen - und ins Gespräch eingreifen. 